\title{
Washout collaterometry: a new method of assessing collaterals using angiographic contrast clearance during coronary occlusion
}

\author{
C Seiler, M Billinger, M Fleisch, B Meier
}

\begin{abstract}
Objective-To investigate the hypothesis that the time to washout of radiographic contrast medium trapped distal to an occluded collateral receiving vessel is inversely related to collateral flow, and that this provides an accurate method for characterising coronary collaterals.

Methods-An intracoronary pressure derived collateral flow index was determined in 54 patients undergoing percutaneous transluminal coronary balloon angioplasty (PTCA). The study group was subdivided according to whether the collateral vessels were sufficient $(n=17)$ or insufficient $(n=37)$ to prevent ECG signs of myocardial ischaemia during PTCA. Washout collaterometry - an angiographic washout method-was carried out simultaneously; after injection of radiographic contrast medium into the collateral receiving vessel followed immediately by vascular occlusion, the number of heart beats was counted until approximately half the length of the epicardial vessel was cleared of contrast.

Results-The collateral flow index was higher $(0.28(0.09) v 0.12(0.07) ; \mathrm{p}<0.0001)$ and the contrast washout time shorter $(8.0$ (2.9) $v 17.5$ (6.7) heart beats; $\mathrm{p}<0.0001)$ in patients with sufficient versus insufficient collaterals. There was an inverse correlation between contrast washout time and collateral flow index $(r=0.72, \mathrm{p}<0.0001)$. Washout of contrast distal to the occluded vessel within 11 heart beats correctly determined sufficient and insufficient collaterals with $88 \%$ sensitivity and $81 \%$ specificity.

Conclusions-Washout collaterometry is a new radiographic contrast washout method based on the inverse relation between collateral flow and the time to clearance of radiographic dye injected into the ipsilateral vessel during PTCA. It appears to be an accurate method of characterising coronary collateral vessels.

(Heart 2001;86:540-546)
\end{abstract}

Keywords: coronary artery disease; collateral circulation; coronary angiography

During the past two decades, evidence has accumulated that the collateral circulation in patients with coronary artery disease is clinically important. Several studies have shown smaller infarcts, less ventricular aneurysm formation, improved ventricular function, and better survival in patients with extensive versus sparse coronary collateral vessels. ${ }^{12}$ In the clinical setting of a non-occluded stenotic coronary artery undergoing balloon angioplasty (percutaneous transluminal coronary angioplasty, PTCA), the measurement of a pressure or velocity derived collateral flow index by means of the angioplasty guide wire distal to the stenosis has been established as a reference method for the quantitative assessment of collaterals. ${ }^{3-5}$ The collateral flow index expresses the amount of flow through collaterals to the area of an occluded vessel as a fraction of the flow through the normally patent vessel.

Doppler or pressure sensor tipped guide wires are established tools for assessing collateral flow in humans but they are quite expensive. An inexpensive but rather crude angiographic method has been employed to qualify the ipsilateral epicardial vascular filling through collaterals from the contralateral side, using a score from 0 to $3 .{ }^{6}$ Several studies have shown that the reliability of this angiographic method can be enhanced if it is performed during PTCA of the collateral recipient area.
This requires double arterial access but allows assessment not only of spontaneously visible but also of recruitable collaterals. ${ }^{7}$

Coronary angiographic video densitometry has been evaluated in patent vessels using the mean transit time of contrast clearance to assess myocardial perfusion, according to the principles of indicator dilution theory. ${ }^{8}$ Based on these considerations, we sought to develop a more exact, inexpensive, and easy to apply angiographic method for characterising coronary collaterals. Our aim in this study was to test the hypothesis that the time to clearance or washout of radiographic contrast medium trapped distal to an occluded collateral receiving vessel is inversely related to collateral flow, and that this provides an accurate method of characterising coronary collaterals (figs 1 and 2).

\section{Methods}

PATIENTS

Fifty four patients (mean (SD) age 62 (10) years; 44 men, 10 women) with one to three vessel coronary artery disease were included in the study. All underwent PTCA of one stenotic lesion because of symptoms related to stable coronary artery disease. Patients were selected prospectively on the basis that they had no previous infarction in the myocardial area undergoing PTCA and no baseline ECG ST 


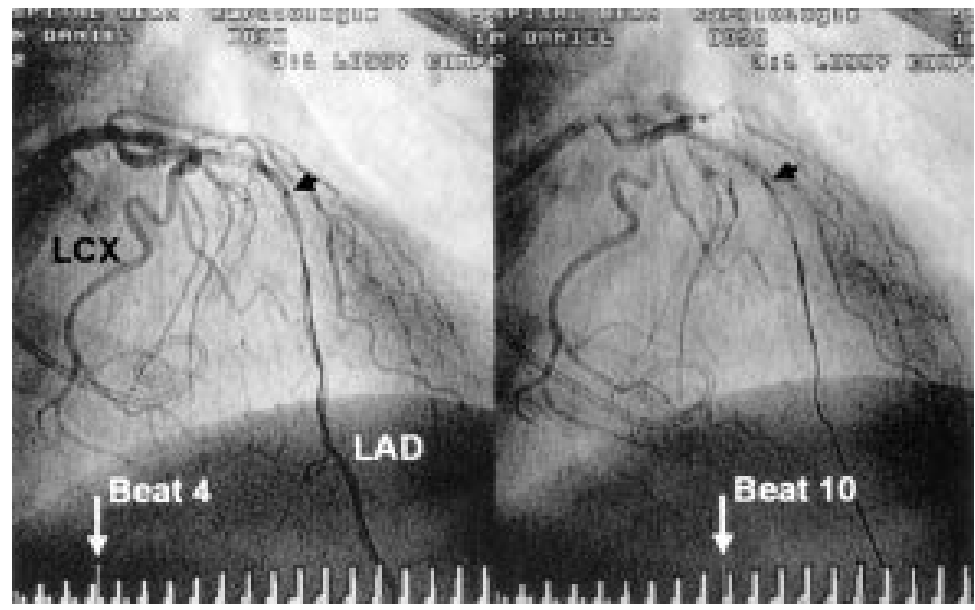

Figure 1 Coronary angiogram (projection: right anterior oblique $17^{\circ}$, cranial $28^{\circ}$ ) showing fast contrast washout in the left anterior descending (LAD) coronary artery. On the left is the situation four heart beats (white arrow) after angioplasty balloon inflation (position in the mid-LAD; black arrow), with the LAD distal to the balloon filled with radiographic contrast medium. On the right is the situation 10 heart beats (white arrow) after angioplasty balloon inflation (position in the mid-LAD; black arrow), with the radiographic contrast medium washed out distal to the balloon. The collateral flow index in this case was 0.37 .

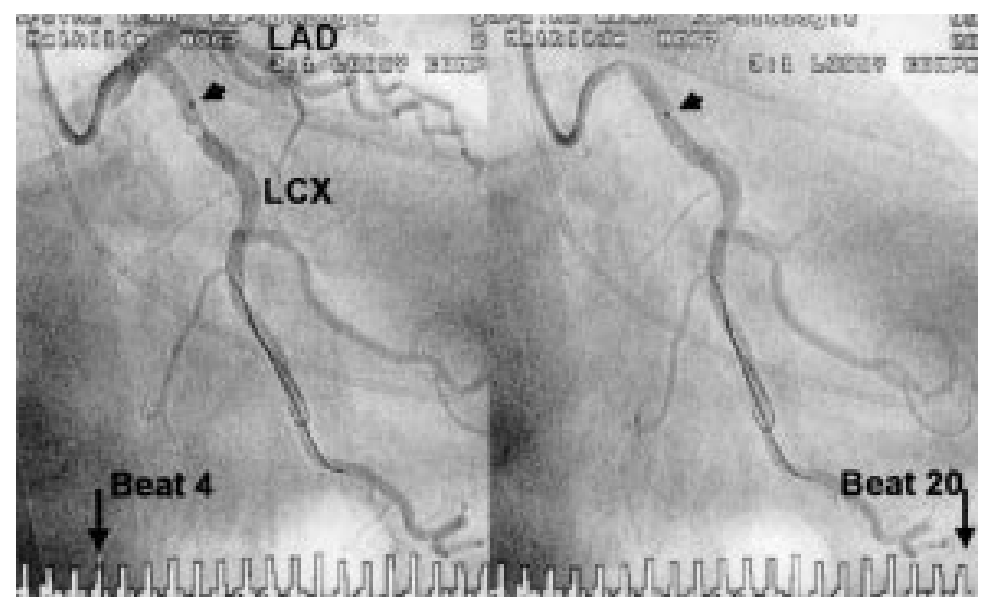

Figure 2 Coronary angiogram (projection: right anterior oblique $27^{\circ}$, caudal $24^{\circ}$ ) showing slow contrast washout in the left circumflex ( $L C X)$ coronary artery. On the left is the situation four heart beats (black, large arrow) after angioplasty balloon inflation (position in the proximal LCx; black arrow), with the LCX distal to the balloon filled with radiographic contrast medium. On the right is the situation 20 heart beats (black, large arrow) after angioplasty balloon inflation, with the radiographic contrast medium not yet washed out distal to the balloon. The collateral flow index in this case was 0.06.

segment abnormalities. The investigation was approved by the institutional ethics committee, and the patients gave informed consent for their participation in the study.

The study population was divided in two groups according to the absence (sufficient collaterals, $\mathrm{n}=17$ ) or presence (insufficient collaterals, $\mathrm{n}=37$ ) of signs of myocardial ischaemia during the first minute of balloon occlusion of the stenosis to be revascularised. Myocardial ischaemia was defined as ST segment changes $>0.1 \mathrm{mV}$ present on an intracoronary ECG lead obtained from the angioplasty guide wire (fig 3 ). ${ }^{45}$

CARDIAC CATHETERISATION AND CORONARY ANGIOGRAPHY

Patients underwent left sided cardiac catheterisation for diagnostic purposes. Aortic pressure was measured using the PTCA guiding catheter. Biplane left ventriculography was performed,

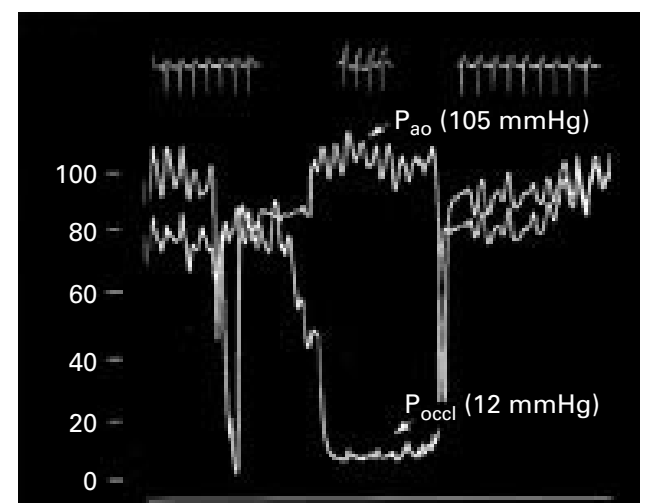

Figure 3 Determination of coronary collateral flow index (CFI) using mean aortic $\left(P_{a d}\right)$ and distal mean coronary wedge pressure $\left(P_{\text {occ }}\right)$ measurements (vertical axis: pressure). The occlusion of the coronary vessel in this case lasted 60 seconds $\left(P_{\text {occl }}=12 \mathrm{~mm} \mathrm{Hg}\right)$. An intracoronary ECG obtained from the pressure guide wire is shown at the top of the panel. During coronary occlusion there were major ST segment elevations, qualifying this patient as one with insufficient collaterals. $C F I=\left(P_{o c l}-C V P\right) /\left(P_{a o}-\right.$ CVP). CVP, central venous pressure, assumed to be $5 \mathrm{~mm}$ $\mathrm{Hg}$.

followed by coronary angiography using ioversol (non-ionic) or ioxaglate (ionic) as radiographic contrast media. Coronary artery stenoses were estimated quantitatively as per cent diameter reduction.

CORONARY COLLATERAL ASSESSMENT

We determined the extent of the angiographic collateral circulation in all patients before PTCA. This was classified on a scale of 0 to 3 , where $0=$ no filling by contrast of the distal vessel through collaterals; $1=$ small side branches filled; $2=$ major side branches of the main vessel filled; and 3 = main epicardial vessel filled. ${ }^{9}$ In 32 patients, this angiographic collateral score was also assessed during occlusion of the stenosed vessel, by injecting radiographic contrast medium through the guiding catheter and through a second coronary catheter into the contralateral collateral supply vessel.

In all study patients, coronary collateral flow relative to normal antegrade flow through the non-occluded coronary artery (the collateral flow index) was determined using intracoronary pressure measurements. This pressure derived collateral flow index has been validated against Doppler derived collateral flow index and intracoronary ECG signs of ischaemia. ${ }^{4}$ In comparison with intracoronary Doppler, the standard error of the estimate (SEE) for the pressure measurements of collateral flow index was 0.08 .

A 0.014 inch $(0.36 \mathrm{~mm})$ fibreoptic pressure monitoring wire (PressureWire, Radi Medical, Uppsala, Sweden) was set at zero, calibrated, advanced through the guiding catheter, and positioned distal to the stenosis to be dilated. The collateral flow index was determined by simultaneous measurement of mean aortic pressure $\left(\mathrm{P}_{\mathrm{ao}}, \mathrm{mm} \mathrm{Hg}\right)$ through the angioplasty guiding catheter and distal coronary artery pressure during balloon occlusion $\left(\mathrm{P}_{\text {occl }}\right.$, $\mathrm{mm} \mathrm{Hg}$ ) (fig 3). Central venous pressure (CVP) was estimated to be $5 \mathrm{~mm} \mathrm{Hg}$. The collateral flow index was calculated as $\left(P_{\text {occl }}-\mathrm{CVP}\right) /\left(\mathrm{P}_{\mathrm{ao}}-\mathrm{CVP}\right) .^{10}$ 
Table 1 Patient characteristics and clinical data

\begin{tabular}{llll}
\hline & $\begin{array}{l}\text { Sufficient } \\
\text { collaterals }\end{array}$ & $\begin{array}{l}\text { Insufficient } \\
\text { collaterals }\end{array}$ & p Value \\
\hline $\mathrm{n}$ & 17 & 37 & $\mathrm{NS}$ \\
Age (years) & $66(9)$ & $58(10)$ & 0.01 \\
Men & 13 & 31 & $\mathrm{NS}$ \\
Women & 4 & 6 & $\mathrm{NS}$ \\
Smoking & 4 & 11 & 0.01 \\
Systemic hypertension & 13 & 14 & $\mathrm{NS}$ \\
Obesity & 2 & 4 & $\mathrm{NS}$ \\
Dyslipidaemia & 11 & 20 & $\mathrm{NS}$ \\
Family history & 4 & 16 & $\mathrm{NS}$ \\
Diabetes mellitus & 2 & 5 & $\mathrm{NS}$ \\
CCS class & $1.9(1.1)$ & $1.8(1.3)$ & $\mathrm{NS}$ \\
Previous infarction & 3 & 7 & $\mathrm{NS}$ \\
Drug treatment & 2 & 10 & 0.05 \\
$\quad$ Calcium antagonist & 7 & 6 & $\mathrm{NS}$ \\
$\quad$ ACE inhibitor & 9 & 22 & $\mathrm{NS}$ \\
$\quad$ Blocker & 5 & 11 & $\mathrm{NS}$ \\
$\quad$ Nitrates & 5 & 16 & $\mathrm{NS}$ \\
$\quad$ Lipid lowering agents & 14 & 35 & $\mathrm{NS}$ \\
$\quad$ Aspirin & 3 & 2 & \\
Diuretics & & &
\end{tabular}

Values are mean (SD) or $\mathrm{n}$.

*In territory not involved in current angioplasty procedure.

ACE, angiotensin converting enzyme; CCS, Canadian Cardiovascular Society class of angina pectoris; sufficient collaterals, no intracoronary ECG ST segment shift ( $>1 \mathrm{~mm}$ ) during percutaneous transluminal coronary angioplasty.

\section{STUDY PROTOCOL}

Following diagnostic coronary angiography, including angiographic collateral assessment, an interval of 10 minutes was allowed for dissipation of the effect of the contrast medium on coronary flow velocity and vasomotion. Intracoronary or oral glyceryl trinitrate was given. The pressure guide wire was positioned distal to the stenosis to be dilated. An intracoronary ECG obtained from the guide wire was recorded continuously. In patients in whom Rentrop's collateral classification ${ }^{6}$ was determined during PTCA, a second coronary catheter was inserted into the contralateral vessel through an additional 5 French sheath introduced into the same femoral artery.

Immediately before PTCA, 10-15 $\mathrm{ml}$ of contrast medium were injected into the ipsilateral vessel. The starting point for assessment of washout by collaterals was announced by the

Table 2 Coronary angiographic and collateral flow data

\begin{tabular}{llll}
\hline & $\begin{array}{l}\text { Sufficient } \\
\text { collaterals }\end{array}$ & $\begin{array}{l}\text { Insufficient } \\
\text { collaterals }\end{array}$ & p Value \\
\hline $\mathrm{n}$ & 17 & 37 & \\
Number of vessels diseased & $1.7(0.7)$ & $1.7(0.6)$ & $\mathrm{NS}$ \\
Diameter stenosis before PTCA & $85(7) \%$ & $79(10) \%$ & 0.04 \\
Coronary artery undergoing PTCA & & & $\mathrm{NS}$ \\
$\quad$ LAD & 9 & 22 & $\mathrm{NS}$ \\
$\quad$ LCx & 5 & 4 & $\mathrm{NS}$ \\
$\quad$ RCA & 5 & 11 & $\mathrm{NS}$ \\
Site of PTCA & 7 & 18 & $\mathrm{NS}$ \\
$\quad$ Proximal third & 5 & 5 & $\mathrm{NS}$ \\
$\quad$ Middle third (\%) & 4 & 14 & 0.001 \\
$\quad$ Distal third & $1.0(0.6)$ & 26 & 0.03 \\
Angina pectoris during PTCA & & $0.6(0.6)$ & \\
Angiographic collateral score before & $1.1(1.2)$ & $0.6(0.7)(\mathrm{n}=19)$ & 0.19 \\
$\quad$ PTCA & $(\mathrm{n}=13)$ & & \\
Angiographic collateral score during & $0.28(0.09)$ & $0.12(0.07)$ & $<0.0001$ \\
$\quad$ PTCA & $8.0(2.9)$ & $17.5(6.7)$ & $<0.0001$ \\
Collateral flow index & $74(12)$ & $70(9.5)$ & $\mathrm{NS}$ \\
Contrast washout time (heart beats) & $65(14) \%$ & $67(10) \%$ & $\mathrm{NS}$ \\
Heart rate (beats/min) & 10 & 27 & $\mathrm{NS}$ \\
LV ejection fraction & 7 & 9 & $\mathrm{NS}$ \\
Ioversol as contrast medium & & & \\
Ioxaglate as contrast medium & & & \\
\hline
\end{tabular}

Values are mean (SD) or $\mathrm{n}$.

$\mathrm{LAD}$, left anterior descending coronary artery; LCx, left circumflex coronary artery; LV, left ventricular; PTCA, percutaneus transluminal coronary angioplasty; RCA, right coronary artery; sufficient collaterals, no intracoronary or surface lead ECG ST segment shift $(>1 \mathrm{~mm})$ during PTCA. operator to the "timekeeper"-that is, the assistant who performed the pressure recordings. Washout of the contrast medium distal to the occluded vessel was filmed at a frame rate of 12.5 images/second. The number of heart beats after balloon inflation was counted simultaneously until approximately half the length of the epicardial vessel distal to the occlusion was cleared of contrast (figs 1 and 2). For off-line analysis, digitally recorded video film was redisplayed in real time.

PTCA was performed using balloon dilatation catheters ranging in diameter from 2.5$4.0 \mathrm{~mm}$. Balloon inflation was maintained for one minute. Measurements were made of $P_{\text {occl }}$ and simultaneous $\mathrm{P}_{\text {aо }}$, along with intracoronary and surface lead ECG recordings (fig 3). Washout collaterometry data analysis was based on the first balloon inflation. In patients in whom Rentrop's collateral classification was determined during PTCA, injection of contrast medium through the contralateral coronary catheter was done during a second occlusion of the stenosis to be dilated.

\section{STATISTICAL ANALYSIS}

Between-group comparisons of continuous demographic, angiographic, haemodynamic, and collateral flow data were made using an unpaired Student $t$ test. A $\chi^{2}$ test was used for comparison of categorical variables among the two study groups. The relation between collateral flow index values and contrast washout time or angiographic collateral score was assessed by linear regression analysis. The accuracy of the washout method compared with an ECG based assessment of the collateral circulation or with continuous collateral flow index values was determined using receiver operating curves. Values are given as mean (SD). Significance was defined at a probability value of $\mathrm{p}<0.05$.

\section{Results}

PATIENT CHARACTERISTICS AND CLINICAL DATA Patients with sufficient collaterals were older than those with insufficient collaterals (table 1). There were no significant differences between the two study groups with respect to the frequency of cardiovascular risk factors (except for systemic hypertension), the use of vasoactive and lipid lowering substances, or the sex of the patients. The Canadian Cardiovascular Society (CCS) angina score and the presence of a previous myocardial infarction in non-PTCA territory were similar in the two study groups.

\section{CORONARY ANGIOGRAPHIC AND COLLATERAL}

\section{FLOW DATA}

The number of vessels affected by coronary artery disease did not differ between the study groups (table 2). Per cent diameter stenosis before PTCA was more severe in patients with sufficient than insufficient collaterals. There were no statistical differences among the study groups regarding the distribution of the target vessels or the site of the stenosis treated by PTCA. Left ventricular ejection fraction was similar in the study groups. Mean (SD) aortic 


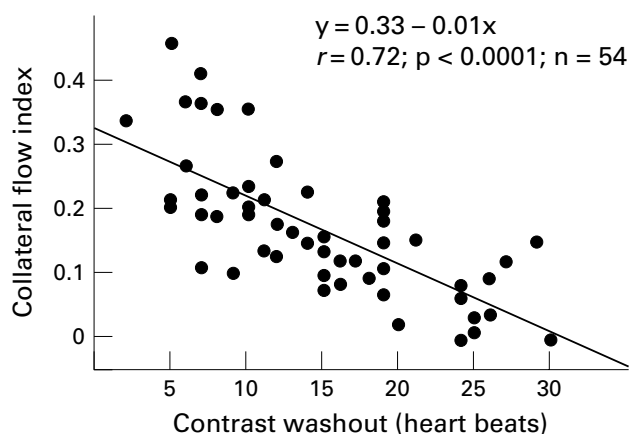

Figure 4 Correlation between occlusive radiographic contrast washout time and collateral flow index. There was a significant inverse linear association between the two variables.

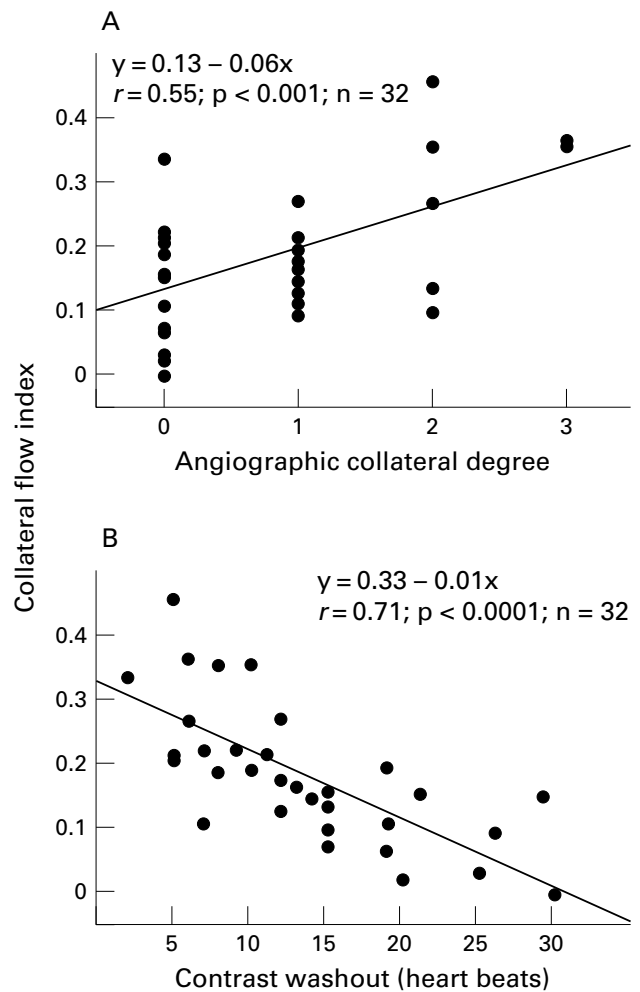

Figure 5 Correlations between collateral flow index and (A) angiographic collateral degree during PTCA, and (B) occlusive radiographic contrast washout time.

pressure was 100 (22) $\mathrm{mm} \mathrm{Hg}$ in the group with sufficient collaterals and 91 (11) $\mathrm{mm} \mathrm{Hg}$ in the group with insufficient collaterals $(p=0.07)$. Left ventricular end diastolic pressure did not differ between patients with sufficient and insufficient collaterals, at 10 (2) $v 9$ (3) $\mathrm{mm} \mathrm{Hg}$ (NS).

The radiographic contrast media ioversol and ioxaglate were used with similar frequency in the two groups (table 2). There were no complications induced by occlusion of the vessels and the simultaneous contrast medium injections.

Qualitative and quantitative variables for the assessment of the collateral circulation were different among the groups, except for the angiographic collateral classification during PTCA (table 2). Patients with sufficient collaterals had angina during PTCA less often than

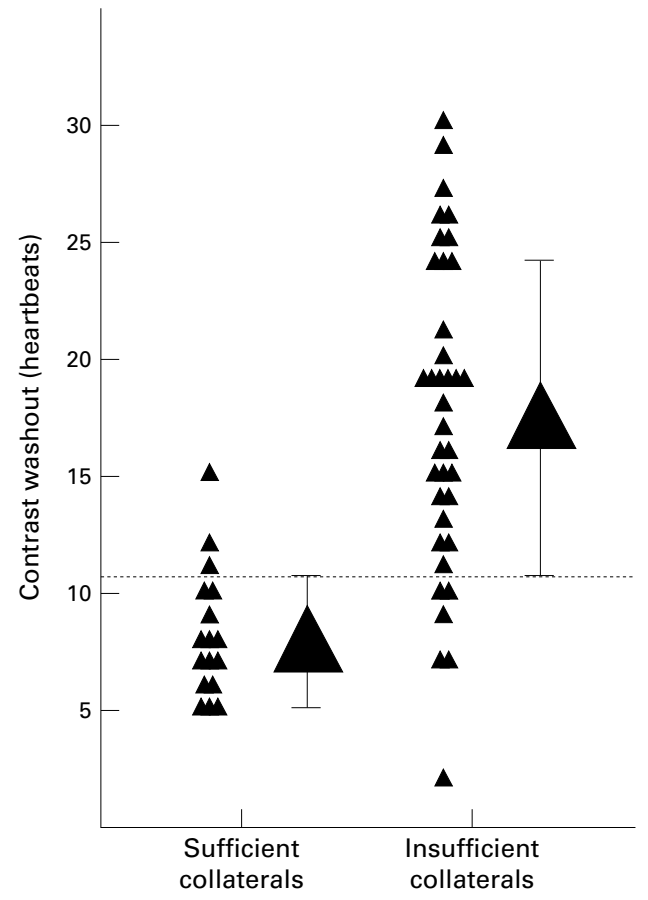

Figure 6 Distinction between collaterals that were sufficient or insufficient to prevent ECG ST segment changes during a one minute coronary occlusion using occlusive radiographic contrast washout time. The large triangles with error bars indicate mean contrast washout time with standard deviations.

those with insufficient collaterals. The angiographic collateral score determined before PTCA was significantly higher in patients with sufficient than in those with insufficient collaterals. Collateral flow index was higher and the time to clearance of contrast medium shorter in patients with sufficient than in those with insufficient collaterals. The variability of heart beats counted by two on-line readers was: $\mathrm{y}=1.1 \times-2.1 ; \mathrm{SEE}=2$ heart beats; $r=0.8$

Figure 4 shows that the washout of contrast medium to about half the initially filled ipsilateral epicardial vessel was inversely related to collateral flow index. The correlation between contrast washout and collateral flow index was closer than the direct relation between the angiographic collateral score obtained during PTCA and the collateral flow index (fig 5). The areas under the receiver operating curve of contrast washout heart beats in relation to ECG based sufficient/insufficient collaterals and in relation to continuous collateral flow index values were 0.90 and 0.89 (for collateral flow index $=0.30$ as the best cut off), respectively. The best cut off threshold of 11 heart beats until washout of the contrast medium to half the initially filled ipsilateral vessel identified patients with sufficient coronary collaterals with $88 \%$ sensitivity and $81 \%$ specificity (fig 6). Detection of a collateral flow index of 0.30 identified collateral sufficiency with $100 \%$ sensitivity and $72 \%$ specificity. An angiographic collateral score of 2 obtained during stenosis occlusion detected sufficient collaterals with $71 \%$ sensitivity and $68 \%$ specificity. An angiographic collateral score of 1 obtained before stenosis occlusion detected sufficient collaterals with $41 \%$ sensitivity and $85 \%$ specificity. 


\section{Discussion}

In this study of washout collaterometry-a new quantitative angiographic method of assessing collaterals by radiographic contrast washout during occlusion-we show that contrast medium trapped distal to the occlusive balloon is cleared more rapidly the higher the collateral flow to the occluded region. Contrast washout within 11 heart beats after balloon inflation is an accurate measure of a collateral supply to the occluded vascular region that is sufficient to prevent ECG signs of myocardial ischaemia.

RELEVANCE OF CORONARY COLLATERAL ASSESSMENT

There are several reasons for characterising collaterals routinely during coronary angiography, and particularly during PTCA. However, the technical difficulty, poor accuracy (for example, when using angina pectoris during PTCA), and cost (particularly with guide wire based pressure or Doppler sensors) of methods that can be used before and during PTCA $^{11}$ have so far discouraged routine assessment of collaterals.

Collateral assessment before PTCA or during the first balloon inflation provides information on the safety of the procedure which may modify the revascularisation strategy. It also permits risk stratification - in addition to the established indices such as the number of diseased vessels and the ejection fraction ${ }^{3}-$ not only with regard to future cardiac events but also concerning the risk of restenosis (higher in cases with extensive collaterals). ${ }^{12}$ Equally importantly, accurate collateral measurement provides additional insights into the pathophysiological aspects of the human collateral circulation, such as alterations in collateral flow during repeated episodes of myocardial ischaemia (collateral recruitment), during sympathetic stimulation, or under treatment with different vasoactive substances. ${ }^{13-16}$ For the clinical investigation of these aspects of human coronary collaterals, as well as for efficacy studies of drugs promoting collateral growth, precise but costly quantitative measurements of pressure/velocity derived collateral flow indices are necessary. ${ }^{17}$ However, risk assessments and semiquantitative pathogenic investigations may employ inexpensive, easy to apply, and reasonably accurate angiographic methods; washout collaterometry comes into this category.

CLINICAL AND HAEMODYNAMIC VARIABLES FOR COLLATERAL ASSESSMENT

The presence of functionally relevant coronary collaterals can be assessed from the presentation of angina. The terms "first effort", "warm up", "first hole angina", "walk through angina", and "second wind" have been recognised for centuries and describe the phenomenon whereby patients with angina are able to continue exertion after their initial pain with few or no symptoms. It has been shown unequivocally that this tolerance of myocardial ischaemia reflects both collateral recruitment and the biochemical phenomenon of ischaemic preconditioning. ${ }^{13}$ The direct demonstration of collateral recruitment-and any sensitive method for the measurement of collateralsrequires a coronary occlusion model, whether natural or artificial (for example, a PTCA model). ${ }^{11}$ Having established by cardiac catheterisation the diagnosis of a naturally occluded coronary artery without transmural myocardial infarction, radioactive myocardial perfusion tracers are, in principle, the appropriate gold standard for non-invasive determination of collateral function (volume flow rate in $\mathrm{ml} / \mathrm{min} / \mathrm{g}$ of tissue). A model simulating naturally occurring atherosclerotic vessel occlusion by balloon inflation during PTCA provides an elegant way of measuring collaterals in patients scheduled for a percutaneous revascularisation procedure. ${ }^{9} 18$ The simplest way of qualifying collaterals in such patients is to ask them whether they get angina during balloon occlusion of the stenotic lesion to be treated.

The use of the ECG for collateral assessment during PTCA requires leads in the relevant myocardial area. The use of an intracoronary ECG recorded through the PTCA guide wire to quantify collaterals was first described in $1985,{ }^{19}$ and has since been confirmed as a sensitive method for collateral assessment. ${ }^{34}$ In our present study, the intracoronary ECG served as one of the reference indices to test the new washout collaterometry technique, the other being intracoronary occlusive perfusion pressure measurement-a haemodynamic method for collateral assessment that can now be regarded as the gold standard (along with occlusive Doppler measurement). ${ }^{45}$ The diagnostic accuracy of a pressure derived collateral flow index of $<0.3$ for detecting ECG signs of myocardial ischaemia during coronary occlusion was similar in both studies: $75 \%$ and $70 \%$ sensitivity, and $92 \%$ and $94 \%$ specificity, respectively. Analyses of more than 450 patients assessed for collaterals by intracoronary pressure or Doppler measurements have shown that a collateral flow index of $25 \%$ is the best cut off value to differentiate patients without and with ECG signs of ischaemia during occlusion; these haemodynamic methods can discriminate between such patients with $71 \%$ sensitivity and $84 \%$ specificity. ${ }^{11}$

\section{CORONARY ANGIOGRAPHIC VARIABLES FOR}

COLLATERAL ASSESSMENT

The angiographic score point system ${ }^{6}$ of retrograde epicardial filling from the collateral supplying vessel has been the most widely used method of collateral assessment in humans because it is always available during coronary angiography. This angiographic assessment of vascular filling through collaterals should be used during coronary occlusion, as this increases the sensitivity of the method by detecting both spontaneously visible and recruitable collaterals. ${ }^{20}$ In our present study, the sensitivity of the traditional angiographic method performed during occlusion for correctly identifying ECG signs of ischaemia was similar to that of pressure derived collateral flow index measurements, but the specificity was considerably lower. However, the major drawback of the 
Rentrop method undertaken during as opposed to before occlusion is its low ratio of practical feasibility to the gain in accuracy. For many interventionalists, the effort of placing a second coronary catheter through an additional introducer for injecting contrast during ipsilateral occlusion is prohibitive. On the other hand, performing the angiographic score point system without ipsilateral occlusion is relatively easy but quite inaccurate.

In contrast, angiographic timing of vascular clearance, or washout, by collaterals is a much easier procedure, and it always provides a measure of recruitable collateral flow as it is done during vessel occlusion. Compared with Rentrop's score point system, the washout method is quantitative as it involves determining either the time or the number of cardiac cycles required to wash out angiographic contrast medium during stenosis occlusion. To enhance the practicality of the method, the time to washout of half the epicardial vessel length distal to the occluded balloon should be determined by counting heart beats while continuously recording the $x$ ray film for off-line analysis. The cut off value of 11 heart beats for contrast washout for distinguishing between collaterals sufficient and insufficient to prevent myocardial ischaemia during coronary occlusion is a convenient and accurate differentiator.

STUDY LIMITATIONS

There are potential drawbacks to the method related to poor coordination between the balloon inflating and contrast injecting operators-for example, too slow or incomplete balloon inflation results in a falsely fast washout, thus impairing the sensitivity of the method as fast contrast clearance may be misinterpreted as reflecting collateral flow.

To minimise the confounding effect of distal ipsilateral microvascular resistance on the washout collaterometry, our study protocol did not involve hyperaemic conditions.

When intracoronary ECG ST segment changes were determined during occlusion (as one of our reference methods), care was taken to perform exactly one minute occlusions in all patients, and to assess the ST segment at the end of the balloon inflation. Non-standardised ST assessment may lead to a misclassification of collateral vessel sufficiency, as ST segment changes require a certain time to develop. Using one minute occlusions, the risk of falsely categorising patients as having sufficient collaterals is higher than the risk of falsely categorising patients as having insufficient collaterals. Because of this possible limitation to the use of the ECG as a reference method, we also used another standard method for deriving the collateral flow index - the aortic and coronary wedge pressure method. However, a potential drawback of pressure measurements is that large ischaemic myocardial territories in patients with few collaterals may result in an overestimation of intracoronary pressures because of increased left ventricular filling pressures (see also table 2).

As shown in fig 4, a further limitation of the washout method is that at the low end of the range of collateral flow indices $(<0.15)$, the method is not sensitive enough to determine collateral flow differences accurately. This insensitivity may in part reflect incorrect counting of heart beats.

Restricted image resolution may affect the outcome of angiography because determination of disappearance always depends not only on the spatial but also on the temporal resolution of the imaging system. However, using a 12.5 frames/second temporal resolution appeared to be sufficient for the washout method, as the relation between collateral flow index and washout time did not change when we doubled the speed to 25 frames/second ( $n=36$; unpublished data). Spatial resolution is much less of an issue in determining washout than when using traditional angiographic grading methods, as the contrast clearance from large vessels is assessed, rather than the retrograde filling of possibly small vessels. Nevertheless, variable patterns of contrast clearance-such as discrete washout or more diffuse fading in contrast brightness, as well as variable distal vessel lengths - may make it difficult to determine the exact time point of washout.

Finally, the size of the study group with sufficient collaterals was relatively small, so that non-significant differences between the groups need to be interpreted with caution.

CONCLUSIONS

Washout collaterometry, a new radiographic contrast clearance method, relies on the fact that the time to washout of radiographic dye injected into the ipsilateral vessel during PTCA is inversely related to collateral flow index. It appears to be an accurate method of characterising coronary collaterals.

Supported by a grant from the Swiss National Science Foundation (No 32-58945.99).

1 Habib GB, Heibig J, Forman SA, et al. Influence of coronary collaterals on myocardial infarct size in humans: results of phase I thrombolysis in myocardial infarction (TIMI) trial. Circulation 1991;83:739-46.

2 Hansen J. Coronary collateral circulation: clinical significance on survival in patients with coronary artery occlusion. Am Heart f 1989;117:290-5.

3 Pijls NHJ, Bech GJW, El Gamal MIH, et al. Quantification of recruitable coronary collateral blood flow in conscious humans and its potential to predict future ischemic events. 7 Am Coll Cardiol 1995;25:1522-8.

4 Seiler C, Fleisch M, Garachemani AR, et al. Coronary collateral quantitation in patients with coronary artery disease using intravascular flow velocity or pressure measurements. F Am Coll Cardiol 1998;32:1272-9.

5 van Liebergen RAM, Piek JJ, Koch KT, et al. Quantification of collateral flow in humans: a comparison of angiographic, of collateral flow in humans: a comparison of angiographic, Coll Cardiol 1999;33:670-7.

6 Rentrop KP, Cohen M, Blanke H, et al. Changes in Rentrop $\mathrm{KP}$, Cohen $\mathrm{M}$, Blanke $\mathrm{H}$, et al. Changes in
collateral channel filling immediately after controlled coronary artery occlusion by an angioplasty in human subjects. F Am Coll Cardiol 1985;5:587-92.

7 Piek JJ, van Liebergen RA, Koch KT, et al. Comparison of collateral vascular responses in the donor and recipient coronary artery during transient coronary occlusion assessed by intracoronary blood flow velocity analysis in patients. F Am Coll Cardiol 1997;29:1528-35.

8 Pijls NH, Uijen GJ, Hoevelaken A, et al. Mean transit time for the assessment of myocardial perfusion by videodensitometry. Circulation 1990;81:1331-40.

9 Rentrop KP, Thornton JC, Feit F, et al. Determinants and protective potential of coronary arterial collaterals as assessed by an angioplasty model. Am 7 Cardiol 1988;61: assessed

10 Pijls NHJ, van Son JAM, Kirkeeide RL, et al. Experimental basis of determining maximum coronary, myocardial, and collateral blood flow by pressure measurements for assessing functional stenosis severity before and after percutaneous coronary angioplasty. Circulation 1993;86:1354-67. 
11 Seiler C, Fleisch M, de Marchi SF, et al. Functional assessment of collaterals in the human coronary circulation. Semin Intervent Cardiol 1998;3:13-20.

12 Wahl A, Billinger M, Fleisch M, et al. Quantitatively assessed coronary collateral circulation and restenosis following percutaneous revascularization. Eur Heart $f$ 2000;21:1776-84.

13 Billinger M, Fleisch M, Eberli FR, et al. Is the development of myocardial tolerance to repeated ischemia in humans due to preconditioning or to collateral recruitment? $\mathcal{F} A m$ Coll Cardiol 1999;33:1027-35.

14 Bache RJ, Foreman B, Hautamaa PV. Response of canine coronary collateral vessels to ergonovine and alphaadrenergic stimulation. Am f Physiol 1991;261:H1019-25.

15 Piek JJ, van Liebergen AM, Koch KT, et al. Pharmacological modulation of the human collateral vascular resistance in acute and chronic coronary occlusion assessed by intracoronary blood flow velocity analysis in an angioplasty model. Circulation 1997;96:106-15.

16 Seiler C, Fleisch M, Billinger M, et al. Simulaneour Seler C, Fleisch M, Billinger M, ot al. Simultaneous intracoronary velocity- and pressure-derived assessment of adenosine-induced collateral hemodynamics in patients with one- to two-vessel coronary artery disease. $7 \mathrm{Am}$ Coll Cardiol 1999;34:1985-94.

17 Seiler C. Human bFGF induces angiogenesis in hen eggs and rat hearts [letter]. Circulation 1999;100:1250-51.

18 Meier B, Lüthy P, Finci L, et al. Coronary wedge pressure in relation to spontaneously visible and recruitable collaterals. Circulation 1987;75:906-13.

19 Meier B, Rutishauser W. Coronary pacing during percutaneous transluminal coronary angioplasty. Circulation 1985; 71:557-61.

20 Cohen M, Sherman W, Rentrop KP, et al. Determinants of collateral filling observed during sudden controlled coronary artery occlusion in human subjects. $f \mathrm{Am}$ Coll Cardiol 1989;13:297-303.

\section{IMAGES IN CARDIOLOGY}

\section{Acute type A aortic dissection in an adult patient with Turner's syndrome}

Acute type A aortic dissection is the most serious cardiovascular complication of Turner's syndrome, and is often fatal. We report a case involving a 38 year old woman with karyotype proven Turner's syndrome ( $46 \mathrm{X}$ iso $\mathrm{X}$ ), who presented with sudden chest pain. An angiographic computed tomographic scan (right) revealed the presence of a type A aortic dissection. Upon hypothermic $\left(28^{\circ} \mathrm{C}\right)$ femorofemoral cardiopulmonary bypass, valvar commissural repair and prosthetic vascular replacement of the ascending aorta were performed with the use of GRF glue. One month later, the patient was asymptomatic.

Cardiovascular malformations are often associated with Turner's syndrome, but aortic dilation and its complications are not so far recognised as being associated with this gonadal dysgenesis. Although the rate of aortic dissection associated with this syndrome is higher than that in the population at large $(0.8$ $\% v 0.01 \%$, respectively), reports in the literature are uncommon. A number of chronic aortic dissections, some acute type $\mathrm{B}$ aortic dissections, and several deaths by intrapericardial aortic rupture have been reported, but only two cases of acute type A aortic dissection with surgical success have been described. Dissections in Turner syndrome are often associated with coarctation, bicuspid aortic valve, and hypertension, but the enlargement of the aorta

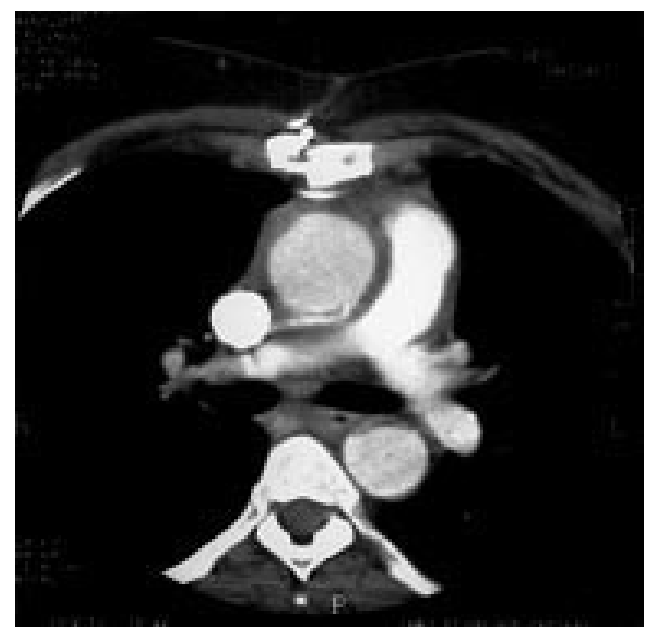

$(6 \%)$ is not recognised as a risk factor. Furthermore, several publications have demonstrated the correlation between aortic dilation and aortic dissection. These results indicate that echocardiographic follow up should be undertaken for all patients with Turner's syndrome in order to avoid this complication by operating on them at the aortic dilation stage.

JEAN-PIERRE MEUNIER
SAED JAZAYERI
MICHEL DAVID

